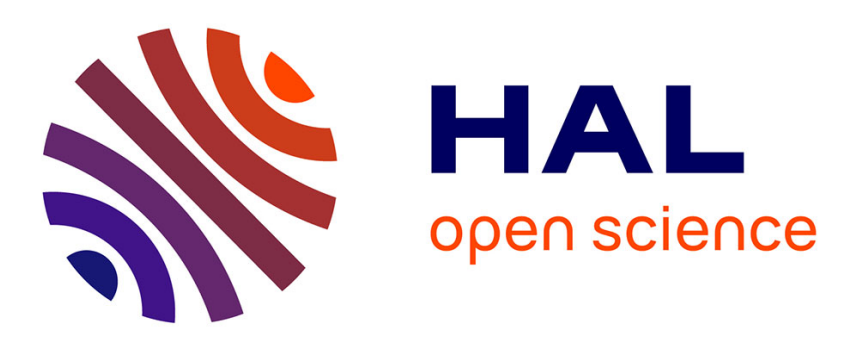

\title{
Prendre en compte les cultures constructives locales pour une meilleure efficacité des projets d'habitat
}

\author{
Thierry Joffroy
}

\section{To cite this version:}

Thierry Joffroy. Prendre en compte les cultures constructives locales pour une meilleure efficacité des projets d'habitat. Un Chronicle, 2016, UN Chronique, Vol III ( $\left.N^{\circ} 3\right)$. hal-01862047

\section{HAL Id: hal-01862047 \\ https://hal.science/hal-01862047}

Submitted on 26 Aug 2018

HAL is a multi-disciplinary open access archive for the deposit and dissemination of scientific research documents, whether they are published or not. The documents may come from teaching and research institutions in France or abroad, or from public or private research centers.
L'archive ouverte pluridisciplinaire HAL, est destinée au dépôt et à la diffusion de documents scientifiques de niveau recherche, publiés ou non, émanant des établissements d'enseignement et de recherche français ou étrangers, des laboratoires publics ou privés. 


\section{Prendre en compte les cultures constructives locales pour une meilleure efficacité des projets d'habitat}

L'étude de l'histoire de la construction montre que les bâtisseurs ont toujours su faire évoluer leur habitat en tirant le meilleur parti des ressources disponibles localement en réponse à leurs besoins et en regard des contraintes économiques, sociales et climatiques locales. Partout dans le monde, des cultures constructives spécifiques ont produit des architectures "contextuelles" correspondant à des modes de construction et d'habiter singuliers. Celles-ci ne sont pourtant pas statiques. Elles sont amenées à évoluer en fonction de nouvelles possibilités offertes, notamment lors d'échanges avec d'autres contrées en matière de savoirs, de savoir-faire et/ou de techniques et matériaux de construction.

Ces cultures constructives locales ont souvent été liées à nécessité reconnue par les sociétés traditionnelles : l'équilibre entre l'homme et la nature qui l'entoure. Cette quête ancienne correspond à ce que l'on qualifie aujourd'hui de "développement durable", plus qu'une nécessité. Que de bons exemples de ce concept sont donc déjà présents à travers le monde! Malheureusement, sous l'influence de la mondialisation, ces intelligences locales tendent à être déconsidérées, et à l'instar d'espèces animales ou végétales, elles sont « en voie de disparition ». II s'agit là non seulement de pertes pour la diversité culturelle de notre monde, mais aussi de pertes de savoirs et de connaissances scientifiques étonnants par leur pertinence et utiles pour l'humanité, à la fois dans la réflexion globale, mais aussi et surtout pour agir localement.

Ces savoirs sont présents à divers niveaux : localisation des établissements humains, répartition des activités et mode de gestion du terroir et des milieux urbains, compositions architecturales, matériaux et organisation de la construction. Ils comprennent aussi des approches extrêmement intéressantes de prévention ou de préparation aux risques et à la reconstruction post-aléas. Dans les milieux fragiles ou à risque fort, les solutions techniques qui leur sont associés sont souvent particulièrement astucieux et typés, ce qui les rend plus faciles à détecter.

Avec la mondialisation, ce sont des pans entiers de l'intelligence humaine en matière de construction qui sont dilapidés, de la même manière et à la même vitesse que disparaissent les grandes forêts de notre planète. Mais cela se fait de façon plus silencieuse alors que les effets en sont tout aussi dévastateurs. Ce changement a commencé dans la première moitié du siècle dernier avec, en toile de fond, l'industrialisation de procédés de construction (essentiellement autour de l'utilisation du ciment et des bétons) et l'universalisation d'une éducation qui leur est exclusivement dédiée (écoles d'ingénieurs, enseignement technique et professionnel).

Aujourd'hui, deux mondes se séparent : celui des « riches », capables d'adopter cette modernité, et celui des « pauvres » qui gardent un mode d'habiter traditionnel, mais qui sous la pression médiatique (et sociale) sont amenés à construire de mauvaises copies ou à procéder à des mélanges technologiques inadaptés, voire même dangereux. Un exemple terrible de ce type de problème a été constaté à Haïti après le séisme de janvier 2010. Ce ne sont pas les petites maisons traditionnelles qui ont tué, mais bel et bien les constructions « modernes » que la grande majorité des haïtiens a du mal à financer accédant finalement à des réalisations se sous-qualité.

Pourtant, les normes de construction internationales continuent de s'imposer, avec un peu partout des résultats similaires. Chaque catastrophe tend à le rappeler. Dans une majorité de cas, l'observation fine montre que les constructions purement traditionnelles résistent mieux ou, tout du moins, entrainent moins de pertes humaines. Et leur remise en état s'avère possible, simple et peu couteuse, donc accessible à beaucoup. Ces modèles devraient donc inspirer les concepteurs des programmes de reconstruction.

C'est dans les années 1970 que se feront les premières prises de conscience, beaucoup inspirées par l'architecte égyptien Hassan Fathy. Quelles alternatives pouvait-on proposer à, d'un côté, des modèles d'habitat « modernes », bien considéré, mais trop cher et, d'un autre côté, des modèles traditionnels, considérés à tort, comme obsolètes. L'approche restera pendant longtemps assez technique. C'est l'époque de la «Technologie Appropriée » qui permit d'accéder à quelques bons résultats, mais dont on en connait aujourd'hui les limites en termes de coût, de reproductibilité et de réponse aux besoins réels.

Malgré cette prise de conscience, l'augmentation des catastrophes et la pression des médias font que les programmes d'habitat privilégient encore trop souvent le quantitatif au qualitatif. Surtout quand les choix doivent être faits dans l'urgence on retombe sur des solutions " universelles ", "prêtes à l'emploi ». Certes, celles-ci peuvent répondre à des besoins à court terme mais elles sont peu efficace à moyen et long terme, voire même contre-productives car mettent en place des standards inadaptés et des espérances impossibles à satisfaire à grande échelle.

Depuis 30 ans, avec divers partenaires, CRAterre-ENSAG mène des recherches en lien avec des programmes sur le terrain, en vue de mieux prendre en compte le potentiel remarquable que des cultures constructives locales. II s'agissait déjà de mieux définir le concept, de trouver des opportunités mise en application sur le terrain, et enfin, de définir des méthodes et stratégies adaptées. Concrètement, l'idée est de repérer les systèmes constructifs, savoir faire et mode d'organisation locaux à l'efficacité éprouvée, puis de les intégrer dans la définition et la conception de programmes et projets, sans écarter de possibles apports des productions industrielles, le tout devant répondre aux besoins et espérances, mais aussi être acceptable socialement et culturellement, et accessible économiquement.

Quelques opérations menées ont plus particulièrement mis en avant la pertinence de tels choix.

A Mayotte, île de l'archipel des Comores, un programme de logement a été lancé au début des années 1980. Ce programme mené par la SIM (Société Immobilière de Mayotte) a bénéficié en préalable d'une étude ethnologique sur l'habitat. Ceci a permit de définir des modèles simple et bien adaptés. L'aide est fournie pour construire un noyau de base qui est ensuite complété par la famille avec ses propres moyens, y compris pour la clôture, les toilettes et la partie agriculture de 
subsistance. Ces trente dernières années, c'est un équivalent de plus de 20000 unités de logements sociaux qui ont ainsi pu être édifiés sur ces bases.

Plus récemment, à Haïti, suite au séisme de janvier 2010, l'ONG Misereor a sollicité CRAterre pour un programme ambitieux d'aide à la reconstruction de 5000 maisons de base en zone rurale et montagneuse. Les constructions traditionnelles, construites en bois avec remplissage en matériaux locaux étant " celles qui n'avaient pas tué ", ont été prises comme base de la réflexion. Leur analyse a révélé des variantes (en fonction des conditions : localisation, altitude, activités économiques, etc...) et l'existence systématique d'un noyau agrandissable. Ces spécificités ont été retenues dans la conception des modèles de maison de base proposés. Suite à l'enthousiasme des populations après la construction des premiers prototypes, le programme à grande échelle a pu être lancé. Il a été réalisé par des ONGs locales, avec les artisans locaux, les «Boss », dans le cadre du système traditionnel d'entraide: le « kombit ». La pratique de décoration de la maison haïtienne a aussi été intégrée. Ainsi, l'architecture aux couleurs vives et gaies a repris sa place devant les maisons en béton, inachevées, grises et tristes, une valorisation de la culture des haïtiens qu'ils ont fortement appréciée.

Ces projets sont des contributions vers la démonstration que l'approche proposée peut donner des résultats efficaces. II s'agit d'investissements utiles et pérennes. Mais en même temps, on constate que le cadre normatif international poursuit son développement. Contrairement aux bonnes intentions affichées, dans les contextes fragiles, celui-ci résulte de plus en plus en de l'exclusion sociale et des pertes irréversibles d'identités locales en matière d'architecture, de mode de vie, et d'aménagement du territoire. Pourtant, le potentiel des cultures constructives locales est énorme et le champ de recherche presqu'infini, vers la mise au point de solutions utiles, esthétiques, et porteuses de résilience et de développement durable.

Dans ces perspectives, il est intéressant de noter que lorsque l'on arrive à renvoyer une image positive de l'architecture traditionnelle aux populations qui en sont détentrices, très vite des sauts qualitatifs peuvent être obtenus et répliqués largement. Pour que cela soit efficace il va falloir poursuivre le travail de fond sur la retro-ingénierie des constructions traditionnelles. En effet, nombre de projets sont bloqués par manque de données techniques à même de rassurer les responsables et bureaux de contrôle sur leurs performances réelles. II s'agit bien ici d'une réflexion sur l'architecture, car c'est souvent dans son intelligence globale que se trouvent les clés la possible valorisation des matériaux locaux, même si ceux-ci ont des performances modestes (terre, bambou, ...). Ceci dit, certaines architectures traditionnelles doivent être améliorées. La réflexion technique reste donc essentielle. Toutefois, c'est bel et bien en valorisant les artisans locaux et en restant proche des solutions qu'ils maitrisent et des coûts de construction qui correspondent à ces pratiques, que l'on a le plus de chances de faire des propositions d'amélioration qui puissent effectivement être adoptées, non seulement dans les projets, mais aussi plus largement, à côté et après les projets afin que le nombre de bénéficiaires, indirects cette fois, soit le plus important possible. .

II est donc urgent que les institutions nationales et internationales prennent conscience de cela. Certaines ont déjà rejoint CRAterre sur ce thème pour lancer un Manifeste pour « Valoriser les cultures constructives locales pour une meilleure efficacité des programmes d'habitat ». Cette initiative est aussi soutenue par UN Habitat dans le cadre de son Global Network for Sustainable Housing (GNSH) et dans certains de ces projets comme au Pakistan, en RDC ou encore en Somalie. L'UNESCO soutient aussi la démarche alors que la Banque mondiale vient elle aussi d'adopter un nouveau cadre (New Environmental and Social Framework) qui reconnait son intérêt. Ceci devrait favoriser son adoption par d'autres organismes, vers une amélioration effective des conditions de vie des populations de notre monde, tout en lui conservant sa diversité culturelle.

Pour faciliter la poursuite de tels processus, dans le cadre de son programme de recherche LABEX AE\&CC, CRAterre collabore avec la FICR pour établir des documents de référence à l'usage des Shelter Cluster qui fournissent des informations de base sur les intelligences des architectures traditionnelles et leurs possibilités de réutilisation dans les programmes de reconstruction. Si bien entendu ces outils s'avèrent utiles, il serait encore mieux de faire un travail de fond dans les pays reconnus à risques de façon à faire plus de travail de façon préventive, permettant ainsi de limiter les dégâts lors des aléas et de renforcer les capacités locales de résilience. Enfin, si ces approches trouvent plus particulièrement leur efficacité dans les zones à risques, il n'en reste pas moins que leur application est tout aussi pertinente dans le cadre de projets d'amélioration de l'habitat, car elle permettent d'identifier les véritables besoins et espérances des communautés locales et de répondre avec des solutions qui leur soient pleinement accessibles, autant des points de vue technique que financier, et qui répondent aussi à la nécessaire reconnaissance sociale de l'habitant. C'est en étant capables de se prendre en charge elles-mêmes, même si elles sont assez démunies, que les communautés peuvent retrouver dignité et confiance en un avenir meilleur.

Thierry Joffroy

Architecte, Chercheur

Président de CRAterre-

Labex AE\&CC / ENSAG

Université Grenoble -Alpes

Plus d'informations et bibliographie disponible sur craterre.org et craterre.hypotheses.org 